\title{
Mechanical Structure and Hardware Circuit Design of Intelligent Vehicle
}

\author{
Xiaoqi Chen * \\ North China Electric Power University, Baoding, 071003, China \\ *www.100xiaohuang@163.com
}

\begin{abstract}
In this paper, the sixth "Freescale" National University Student intelligent motor racing as the background, "based on culture, participation, encourage the exploration, the pursuit of excellence," the guiding ideology, self-designed and fabricated to guide the intelligent vehicle based on electromagnetic system, and in this study based on a realistic path to take excellent program. Environment and its superior ability to adapt to a wide range of expected future applications. This article describes in detail the mechanical structure of the intelligent vehicle design, hardware design to provide some experience for participants.
\end{abstract}

Keywords: Smart car, PID control, sensors, the path to take prio.

\section{Mechanical Structure Design}

In the intelligent car race, the main content of the race is speed, and the mechanical structure of the model car is undoubtedly one of the key factors affecting speed. For this reason, we have done a lot of work on the mechanical structure of the model car, made a lot of adjustments, and achieved satisfactory results.

\subsection{Assembly and Transformation of Car Models}

\subsubsection{Model Assembly}

The assembling of the model car looks simply, but it requires a lot of patience and experience. First, read the instructions carefully. By reading the assembly diagram of the model car, we can understand the usage and installation sequence of different parts. Then, the smart car model is assembled according to the assembly diagram of the model car. According to experience, not only the assembly sequence of the model car should be paid attention to, but also the parts of the model car should be small, so the parts should be prevented from slipping and losing in the assembly process. Especially, since most parts of the model car are made of plastic, extra care should be taken in screw twisting and parts processing to avoid damage.

\subsubsection{Adjustment of Front Wheel Alignment}

During the debugging, we find that the load of steering gear will increase with the increase of steering angle when the model car is turning. In order to reduce steering load as much as possible, we adjusted the front wheel alignment. The function of front wheel alignment is to ensure the stability of straight-line driving, steering lightly and reduce tire wear. Front wheel alignment parameters mainly include: kingpin tilt angle, kingpin tilt angle, front wheel tilt angle and front wheel harness. The kingpin caster angle is the angle between the main pin axis and the ground vertical line in the longitudinal plane of the vehicle. The kingpin inclination angle is the angle between the main pin axis and the ground vertical line in the transverse section of the vehicle. The camber angle of the front wheel is the angle between the horizontal plane of the vehicle and the vertical line of the subgrade and the surface of the wheel. In general, the tilt angle is 0-3 degrees, the tilt angle is 0-10 degrees, the front wheel tilt angle is $0-1$ degrees. In this model, excessive tilt makes the model car steer heavily, so that steering has serious lag, so the kingpin tilt angle is adjusted to 0 degrees in the model car. The front wheel camber and front wheel toe are set at 0 degrees and $0 \mathrm{~mm}$ respectively.

\subsubsection{Differential Adjustment}

The differential speed of the model car has a great influence on turning, and bad differential speed will lead to idling of the rear wheel and side slip. By means of adding thrust bearing and lubricating 
oil, we improve the differential device, which greatly reduces the friction between the right wheel and the rear axle when the model car is turning, thus improving the differential effect and improving the steering performance of the car.

\subsubsection{Adjustment of Steering Arm}

Compared with the processing speed of S12 single chip microcomputer, the response of the steering gear has a great delay, so it is necessary to reform the steering gear. Under the same steering speed, the steering wheel changes more quickly when the connecting point of steering link at one end of the steering gear is farther away from the axis of the steering gear. The front wheel is more sensitive when turning.

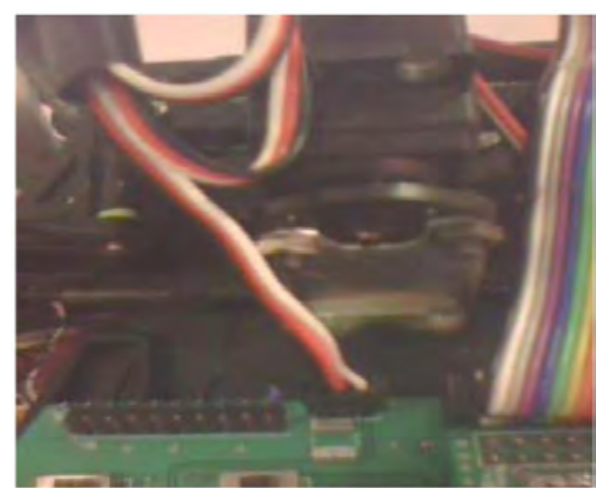

Figure 1. Modification of steering gear

\subsection{Installation of Inductance Coil}

Considering the characteristics of "vertical" and "horizontal" inductance coils, six horizontal inductance coils with uniform horizontal arrangement are selected, which can not only meet the requirements of accurate detection of road information, but also reduce the weight of the sensor as a whole. Two "vertical" inductor coils are arranged symmetrically near the edge to avoid the zeroreturn characteristic. The detailed layout of "vertical" and "horizontal" coils is shown in Figure 1.2. Their respective characteristics are shown in Figure 1.3. There are several factors to consider in the erection of model car sensors:

1. Make sure the inductance coils are symmetrical. If the center of the arrangement is not in the center, and the processing program is in the center of the output of the steering gear, this will lead to the model car on the straight road will also have the problem of swinging left and right.

2.The height of the inductance coil should be high enough to make the model car get enough road information. For the "vertical" inductance coil, too close to the runway cannot reflect the forwardlooking characteristics.

3.The erection of inductance coil must be fixed and unchanged, because the corresponding debugging program is based on the attenuation characteristics of inductance coil to judge the road information, and the inductance of different heights has different attenuation characteristics. This is conducive to continuity and modification of programs.

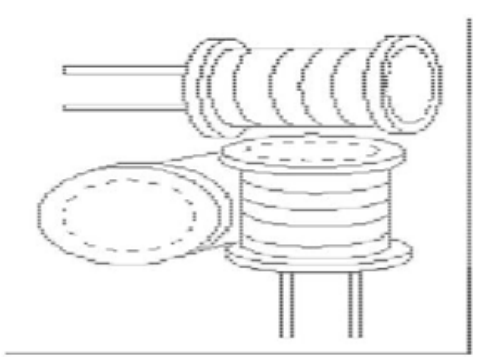

Figure 2. vertical and horizontal layout plan

4. The inclined inductance coil is very strict to the direction, because the angle difference will lead to the coil itself data characteristics change, but the data is asymmetric, resulting in inaccurate control. 


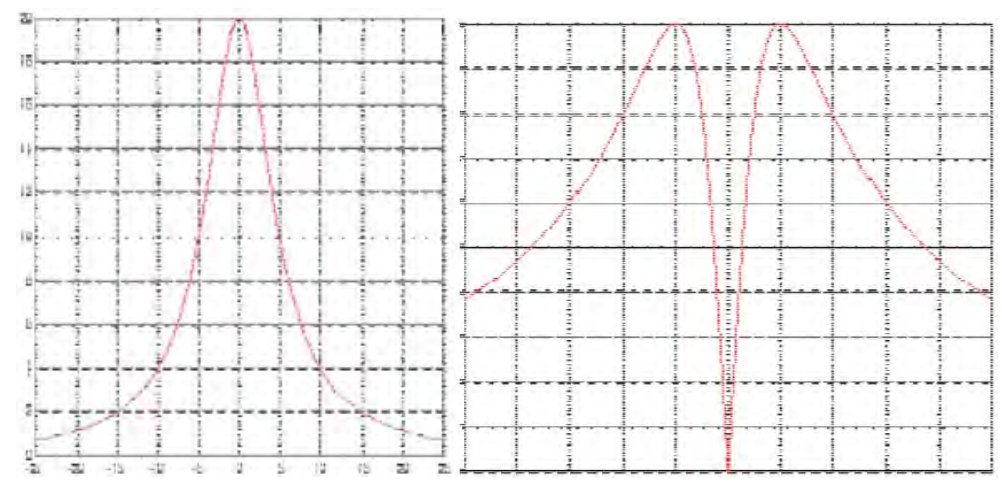

Figure 3. vertical and horizontal data characteristics

\subsection{Installation of Grating Encoder}

For the installation of the grating encoder, the grating disc can be mounted on the motor shaft, and the vehicle speed can be obtained by calculating the motor speed first and then calculating the rear drive shaft of the model vehicle. However, this method is too troublesome, and the grating on the motor shaft will affect the performance of the motor. Therefore, we installed the grating disk on the compass of the rear drive shaft of the model car, and calculated the rear wheel speed of the model car at different times according to the output pulse of the photoelectric sensor.

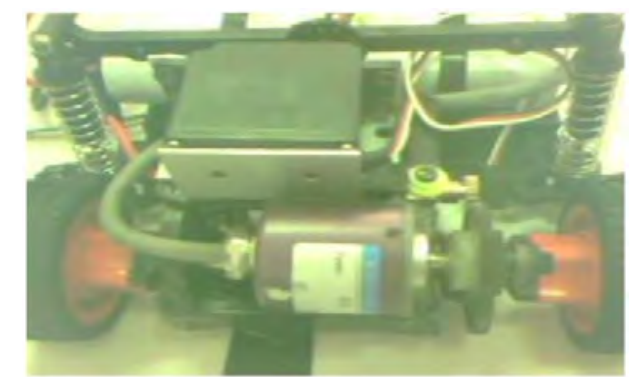

Figure 4. installation of grating encoder

\subsection{Fixing and Installation of Circuit Board}

When designing, considering the layout of the chassis and the layout of the circuit board, selectively perforating the circuit board. The circuit board should be as horizontal as possible and higher than the shock absorber coil to avoid short circuit.

\section{Power and Circuit Design}

The design of the circuit is the foundation of the automatic controller. Intelligent Vehicle Competition designated Freescale S12 series 16-bit MC9S12XS128 as the core control processor. The smallest system is designed on the basis of MC9S12EVKC which is provided by the Organizing Committee. Various interface circuit boards are added to make up the whole circuit system.

\subsection{Power Supply Module}

The power module provides the power needed for other modules of the system. In the design, besides the basic parameters such as voltage range and current capacity, the power conversion efficiency, noise reduction, interference prevention and circuit simplicity should be optimized. Reliable power supply scheme is the foundation of stable and reliable operation of the whole hardware circuit. The power supply of all hardware circuits is supplied by standard 7.2V 2000mAh Ni-cd batteries. Because the working voltage and current capacity of different circuit modules in the circuit are different, the power module should include multiple voltage stabilizing circuits to convert the charging battery voltage into the required voltage of each module. It mainly includes the following different voltages. 
$\mathrm{Z} 5 \mathrm{~V}$ voltage. Mainly for the single-chip system, signal conditioning circuit and part of the interface circuit to provide power supply, voltage stability, low noise, current capacity greater than $500 \mathrm{~mA}$.

$\mathrm{Z} 5 \mathrm{~V}(\mathrm{GD})$ voltage, mainly for the sensor to provide power, this is mainly to achieve the purpose of not affecting the normal operation of the microcontroller.

$\mathrm{Z} 7.2 \mathrm{~V}$ voltage. This part is directly taken from the voltage at both ends of the battery, mainly for the steering gear, rear wheel motor drive module and part of the interface circuit to provide power. Except that $7.2 \mathrm{~V}$ voltage can be obtained directly from the battery, $5 \mathrm{~V}$ voltage needs to be obtained through the step-down voltage-stabilizing circuit, and $12 \mathrm{~V}$ voltage through the step-up voltagestabilizing circuit. The power supply of the motor drive circuit can directly use the voltage at both ends of the battery. The model car often produces a large impact current during the start-up process, on the one hand, it will cause electromagnetic interference to other circuits; on the other hand, the voltage at both ends of the battery will drop due to the internal resistance of the battery, even lower than the minimum voltage required by the voltage regulator circuit, resulting in the reset phenomenon of the single chip microcomputer. In order to overcome the impact of starting impulse current, the electrolytic capacitor with large capacitance can be added to the power supply, and the motor can be controlled by slow starting mode. When starting, the output voltage of the driving circuit has a gradual change process, which makes the starting speed of the motor slightly lower and thus reduces the amplitude of the starting impulse current.

\subsection{Design of Stepdown Voltage Regulator Circuit}

The buck regulator chip we use is LM1117-5.0. LM1117-5.0 is a low-voltage linear regulator with a maximum output current of $1 \mathrm{~A}$, sufficient to provide the required power for $5 \mathrm{~V}$ devices in the system. In addition, its output voltage fluctuation range is only $+0.1 \mathrm{~V}$, and the precision is high. The experiment proves that it can meet the requirements of the intelligent vehicle system. The typical circuit is shown in Figure 5. The ultimate step-down and voltage-stabilizing circuit we used is to add a filter capacitor of $1000 \mathrm{mu} F$ to the output of the circuit on the basis of Figure 5.

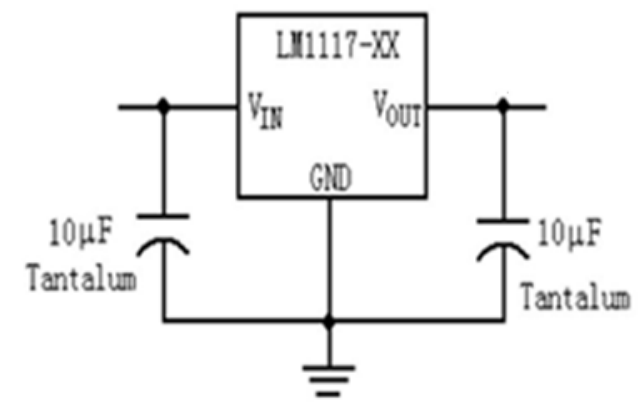

Figure 5. typical circuit diagram of LM1117

If the main regulator chip is TPS7350. At the same time, the internal reset monitoring chip is integrated, and its 5V voltage stabilizing effect is better than that of LM2940. The maximum output current is $2 \mathrm{~A}$, which is enough for single chip microcomputer. Its schematic diagram is shown in Figure 6.

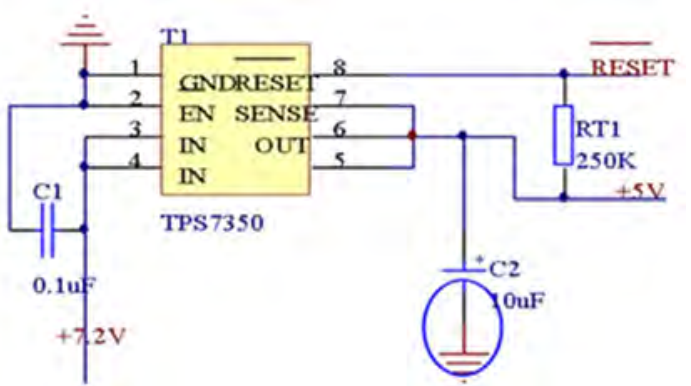

Figure 6. typical circuit of TPS7350 


\section{References}

[1]. Q. Zhuo, K. S. Huang, B.B. shao, learning to build smart cars -- challenging the "flying Carle" cup (Beihang University press, Beijing,2007).

[2]. B.B. shao, On line development method of embedded application of single chip microcomputer (tsinghua university press, Beijing,2004).

[3]. J.M. Zhou, S.M. Zhou, J.H. Peng, Sensor technology and Application (central South University Press, Changsha,2005).

[4]. Ramon Pallas-Areny, John G Webster, Sensors and signal conditioning (tsinghua university press, Beijing,2003).

[5]. G.H. Zong, Creativity and practice of robots (Beihang University press, Beijing,2004).

[6]. Z.S. Yu, Automobile theory (Machinery Industry Press, Beijing, 2004).

[7]. J.Y. Xie, Q. Jia, Microcomputer control technology (National Defense Industry Press, Beijing, 2004). 